\title{
Our dynamic sun: 2017 Hannes Alfvén Medal lecture at the EGU
}

\author{
Eric Priest ${ }^{1}$ ** \\ ${ }^{1}$ Mathematics Institute, St Andrews University, St Andrews KY16 9SS, UK \\ * Invited contribution by Eric Priest, recipient of the EGU Hannes Alfvén Medal for Scientists 2017
}

Correspondence to: Eric Priest (erp@st-andrews.ac.uk)

Received: 15 May 2017 - Accepted: 12 June 2017 - Published: 14 July 2017

\begin{abstract}
This lecture summarises how our understanding of many aspects of the Sun has been revolutionised over the past few years by new observations and models. Much of the dynamic behaviour of the Sun is driven by the magnetic field since, in the outer atmosphere, it represents the largest source of energy by far.

The interior of the Sun possesses a strong shear layer at the base of the convection zone, where sunspot magnetic fields are generated. A small-scale dynamo may also be operating near the surface of the Sun, generating magnetic fields that thread the lowest layer of the solar atmosphere, the turbulent photosphere. Above the photosphere lies the highly dynamic fine-scale chromosphere, and beyond that is the rare corona at high temperatures exceeding 1 million degrees K. Possible magnetic mechanisms for heating the corona and driving the solar wind (two intriguing and unsolved puzzles) are described.

Other puzzles include the structure of giant flux ropes, known as prominences, which have complex fine structure. Occasionally, they erupt and produce huge ejections of mass and magnetic fields (coronal mass ejections), which can disrupt the space environment of the Earth. When such eruptions originate in active regions around sunspots, they are also associated with solar flares, in which magnetic energy is converted to kinetic energy, heat and fast-particle energy. A new theory will be presented for the origin of the twist that is observed in erupting prominences and for the nature of reconnection in the rise phase of an eruptive flare or coronal mass ejection.
\end{abstract}

Keywords. Solar physics astrophysics astronomy (corona and transition region)

\section{Introduction}

Thank you most warmly for the award of the Alfvén Medal, which gives me great pleasure. In addition to a feeling of surprise, I am also deeply humbled, because Alfvén was one of the greats in the field and one of my heroes as a young researcher (Fig. 1). We need dedicated scientists who follow a specialised topic with tenacity and who complement one another in our amazingly diverse field; but we also need time and space for creativity in this busy world, especially for those princes of creativity, mavericks like Alfvén who can view our field widely and blaze trails in new and unexpected directions.

The Sun, an object of worship for early civilisations, is the main source of light and life on Earth and of our space weather with many subtle effects on our environment. It is also a key for astronomy, since many of the fundamental cosmic plasma processes taking place in stars, galaxies and accretion discs can be viewed in much greater detail on the Sun. However, solar physics is at present in a vibrant state since many of the basic properties of the Sun remain a mystery. Although we have made huge progress on them over the past 10 years, definitive answers have not yet been given about how the magnetic field is generated, how the dynamic finescale structure of the atmosphere is created, how the corona is heated and the solar wind accelerated, how prominences are formed and structured, how eruptions of coronal mass ejections are initiated and how reconnection converts energy in solar flares.

These puzzling features are caused by the magnetic field. The Sun is plasma rather than normal gas, so it is coupled in an intimate and subtle way to the magnetic field. This interaction is described by the equations of magnetohydrodynamics (or MHD). To me these are the most beautiful equations I know and are continually revealing new properties. They 


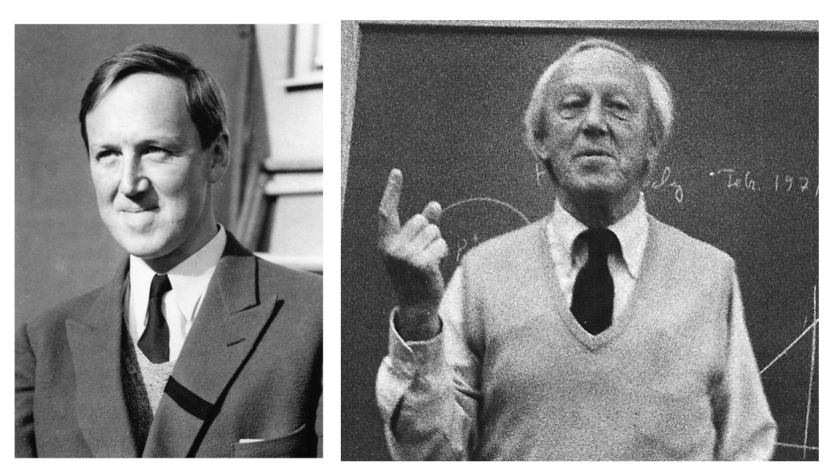

Figure 1. Images of Hannes Alfvén as a young man (age 34) and older.

consist of equations of induction, mass continuity, motion, perfect gas and energy, which take the following form in the corona:

$$
\begin{aligned}
& \frac{\partial \boldsymbol{B}}{\partial t}=\nabla \times(\boldsymbol{v} \times \boldsymbol{B})+\eta \nabla^{2} \boldsymbol{B}, \\
& \frac{\mathrm{d} \rho}{\mathrm{d} t}+\rho \nabla \cdot \boldsymbol{v}=0, \\
& \rho \frac{\mathrm{d} \boldsymbol{v}}{\mathrm{d} t}=-\nabla p+\boldsymbol{j} \times \boldsymbol{B}+\boldsymbol{F}, \\
& p=\frac{k_{B}}{m} \rho T\left(=\frac{\widetilde{R}}{\widetilde{\mu}} \rho T\right), \\
& \frac{\rho^{\gamma}}{\gamma-1} \frac{\mathrm{d}}{\mathrm{d} t}\left(\frac{p}{\rho^{\gamma}}\right)=-\nabla \cdot \boldsymbol{q}-L_{\mathrm{r}}+j^{2} / \sigma+F_{\mathrm{H}},
\end{aligned}
$$

where $\boldsymbol{F}$ includes extra forces, $\boldsymbol{q}$ is the heat flux vector, $L_{\mathrm{r}}$ is the optically thin radiative loss function and $F_{\mathrm{H}}=\rho \epsilon+H_{v}+H_{c}$ is a heating term. These equations are, in general, coupled and serve to determine the primary variables, the plasma velocity $(\boldsymbol{v})$, the magnetic field $(\boldsymbol{B})$, the plasma pressure $(p)$, the plasma density $(\rho)$ and the plasma temperature $(T)$. In addition, the secondary variables, the electric current $(\boldsymbol{j})$ and electric field $(\boldsymbol{E})$, are given explicitly in terms of the velocity $(\boldsymbol{v})$ and magnetic field $(\boldsymbol{B})$ by

$\boldsymbol{j}=\boldsymbol{\nabla} \times \boldsymbol{B} / \mu$,

$\boldsymbol{E}=-\boldsymbol{v} \times \boldsymbol{B}+\boldsymbol{j} / \sigma$,

while $\boldsymbol{B}$ is subject to the condition

$\nabla \cdot B=0$.

This last equation plays the role of an initial condition for the time-dependent Eqs. (1)-(5), which form a complete set, representing nine equations for nine variables. The divergence of Eq. (1) shows that if $\boldsymbol{\nabla} \cdot \boldsymbol{B}$ vanishes initially, then it continues to vanish for all time.

Alfvén (1908-1995) was an early pioneer in proposing MHD ideas, such as frozen flux and, in 1942, MHD waves. In 1937 he suggested that the universe is not filled with a vacuum but is pervaded instead by plasma, which could carry currents that generate a galactic magnetic field. He was trained as an electrical engineer and obtained a $\mathrm{PhD}$ from Uppsala in ultra-short electromagnetic waves (1934). He taught in Uppsala and Stockholm and was appointed a professor at the Royal Institute of Technology in Stockholm in 1940. Later, in 1967, he moved to San Diego, California. He was awarded the 1970 Nobel Prize in physics for "his contributions and fundamental discoveries in magnetohydrodynamics, and their fruitful applications to different areas of plasma physics".

The history of ideas is often fascinating. In particular, Southwood (2015) has reviewed the history of our understanding of the aurora. At the beginning of the last century, Kristian Birkeland, in a ground-breaking move, painstakingly set up polar stations for auroral observations and deduced that the aurora are too high $(100 \mathrm{~km})$ to be atmospheric phenomena. He suggested that they are produced by horizontal electric currents flowing in the ionosphere and closed by currents that flow in on one side along the magnetic field and out on the other (Birkeland, 1908). These are now known as Birkeland currents, but they were only confirmed in 1967. Birkeland thought that the charged particles came directly from the Sun. However, for half a century, the idea of fieldaligned currents was ignored by most solar terrestrial scientists, who were split into two antagonistic schools, one Scandinavian and the other British. The antagonism and ill feeling between these schools was in my view highly regrettable, since it would have been better to listen respectfully to each other by trying to understand the other point of view and remain on good terms. The dominant British school was led by Chapman, who produced a different theory of geomagnetic storms (Chapman and Ferraro, 1930) based on the idea of a closed magnetosphere separated from the interplanetary medium by a magnetopause. Alfvén, however, doggedly kept Birkeland's ideas alive by suggesting that a magnetised particle stream simply flows onto the Earth's magnetic field and is driven by a voltage that comes from the Earth's rotation. Neither picture was correct, but both had elements of truth. They were ultimately unified when Dungey (1961) produced his open-field model of the magnetosphere with the solar wind driving southward-directed magnetic field lines to reconnect at the nose of the magnetosphere and also in its tail. It is this reconnection process that drives field-aligned currents in on the dawn side and out on the dusk side.

Alfvén wrote a highly insightful book in 1950 entitled "Cosmical Electrodynamics" with introductory chapters on charged particle motion, electrical discharges and Alfvén waves propagating along a magnetic field, followed by application chapters on solar physics, magnetic storms and aurora, and cosmic rays. I recently reread the book and was struck by his many imaginative ideas based on the limited observations of the time, but also by how much the newer observations have revolutionised our understanding since then and made many of Alfvén's ideas inapplicable. For exam- 
ple, he thought that the core of the Sun possesses a uniform magnetic field and is surrounded by a global bipolar field that produces a weak field of $20 \mathrm{G}$ at the solar surface. In his mind, the solar interior possesses two turbulent regions, the first near the surface only a few granules thick and the second in the energy-generating core; the core turbulence generated Alfvén waves in the form of very strong flux rings lying in lines of latitude which propagate out along the bipolar field and create a pair of sunspots when a ring reaches the solar surface.

We now know that the Sun is a ball of plasma held together by gravity with a radius of $700 \mathrm{Mm}$ (i.e., 700 million m) compared with the Earth's radius of $6 \mathrm{Mm}$. Its interior possesses a core at which the energy is released by thermonuclear reactions and diffuses very slowly outwards. In the outer onethird of the interior the temperature gradient is large enough to drive convective instability, and this produces a turbulent convection zone. The atmosphere consists of three parts: the thin photosphere with a temperature of $6000 \mathrm{~K}$, from which comes most of the light we see. Above it lies the warmer and rarer chromosphere with a temperature of about $10000 \mathrm{~K}$. Beyond that the outer atmosphere or corona stretches out to fill the heliosphere.

In this lecture I plan to give you some highlights of the progress made since Alfvén's Nobel Prize in 1970 that have come from ground-based and space observations and also from theory. These will include accounts of the photosphere, chromosphere, prominences, coronal heating, solar wind and finally solar flares and coronal mass ejections. Each of these are huge subjects which have spawned many books and reviews, so my discussion will necessarily be simple and selective.

\section{The solar interior}

In 1970, the supposed internal temperature $T(r)$ of the Sun was based purely on theory. The magnetic field of sunspots was generated by a dynamo and rose by magnetic buoyancy. Now there are magnificent computational experiments for the details of how the magnetic flux tubes rise through the solar surface and interact with the overlying magnetic field (e.g., Archontis et al., 2014; Archontis and Hansteen, 2014; Martínez-Sykora et al., 2015; Hansteen et al., 2017).

Now we also know that the Sun oscillates in many different normal modes of vibration. Several million modes have been discovered and form the basis for the new field of solar seismology, from which the internal temperature has been deduced (Christensen-Dalsgaard et al., 1985). The internal rotation of the Sun has been deduced and compared with the surface rotation pattern in which the surface equatorial regions rotate faster than the polar ones. It had been thought that the internal rotation would be constant on cylinders, with the outer cylinders rotating faster than the inner ones; in ad-

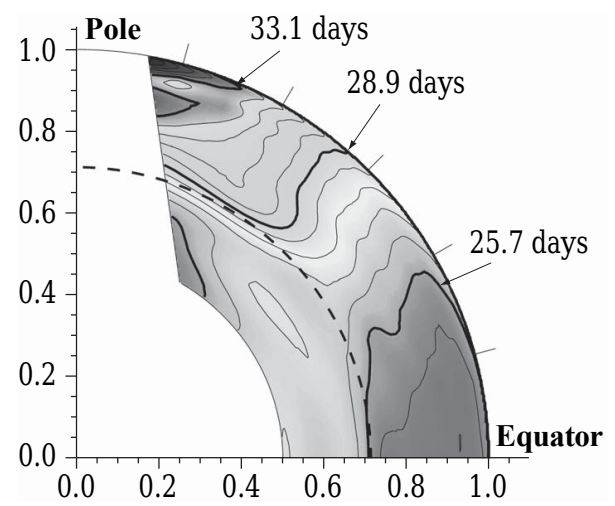

Figure 2. A meridional cut through the solar interior, showing the rotation rate deduced by solar seismology techniques. Rotation times are indicated on three thick curves. There are shear layers at the solar surface and at the tachocline (after Schou et al., 1998).

dition it was expected that the magnetic field would be generated throughout the convection zone.

A huge surprise, therefore, was the discovery from solar seismology that, throughout the convection zone, the rotation is instead constant on cones (Duvall and Harvey, 1984; Duvall et al., 1984). Also, at the base of the convection zone there is an intense shear layer, called the tachocline, which is now thought to be the location of the main dynamo where the magnetic field of sunspots and active regions is generated (Fig. 2). Near the solar surface, one can also see a shear layer, which may possibly be the location of a small-scale dynamo responsible for the small-scale magnetic flux that appears at the solar surface as ephemeral regions, network flux and inner network flux.

\section{The low atmosphere}

\subsection{The photosphere}

The photosphere has a temperature of $6000 \mathrm{~K}$ and is covered with turbulent convection cells on two main scales. Granulation consists of tiny cells with a typical size of $1 \mathrm{Mm}$, whereas supergranulation is much larger with scales of 15$30 \mathrm{Mm}$.

Maps of the line-of-sight magnetic field reveal bipolar $a c$ tive regions around sunspots forming two bands, one north of the equator and the other south (Fig. 3). In 1970, Alfvén thought there was a weak large-scale bipolar magnetic field outside active regions; but by the 1990 s, very strong smallscale magnetic fields had been discovered at the edges and junctions of supergranules, forming a so-called network.

Now, if we map the photospheric magnetic field above a threshold of $500 \mathrm{G}$, the network flux shows up clearly at the edges of supergranules. However, if the threshold is lowered to $25 \mathrm{G}$, far more magnetic flux shows up and tiny elements of a strong magnetic field are revealed at the edges of 


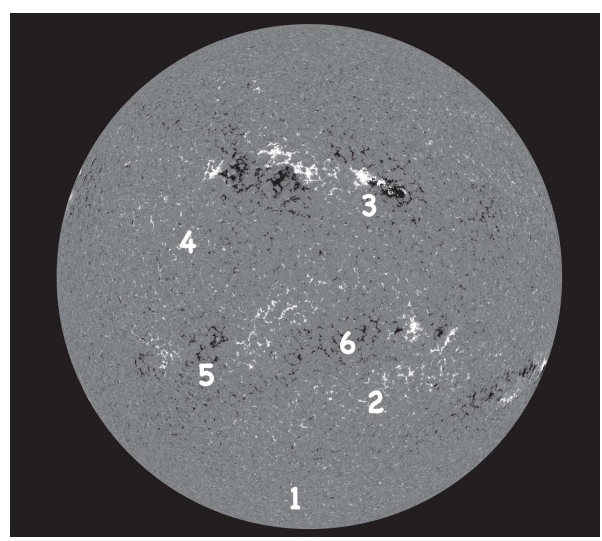

Figure 3. A typical map of the line-of-sight magnetic field in the solar photosphere. The bright regions represent the magnetic field that is directed towards you, while the dark regions have magnetic fields pointing away. The numbers indicate (1) the polar field, (2) a largescale unipolar field, (3) an active region, (4) a tiny bipolar region (called an ephemeral region and a normal network), (5) a remnant active region (6) and an enhanced network field. The two sunspot bands north and south of the equator can clearly be seen (from the HMI instrument on the Solar Dynamics Observatory, SDO).

granules. These elements represent tiny magnetic flux tubes poking through the photosphere and spreading out into the chromosphere and the corona. The result is that the chromospheric and coronal magnetic field is much more complex than we once thought.

Images of the photospheric magnetic field with the highest spatial resolution have come from the wonderful Sunrise balloon mission, which has been able to resolve the kilogauss flux outside active regions for the first time and has a resolution of $100 \mathrm{~km}$ (Solanki et al., 2010; Solanki, 2017; Solanki et al., 2017). An example is shown in Fig. 4, which is compared with the previous highest resolution from the Hinode mission.

Sunspots are locations of huge magnetic flux tubes with magnetic fields generated at the base of the convection zone that then rise through the convection zone by so-called magnetic buoyancy. The flux tubes give rise to sunspots when they break through the solar surface, and they are dark because they are cooler than the surrounding photosphere. Granulation mixes up the surface layers with hotter layers just below the surface and tends to make the surface hotter and brighter than it would otherwise be. Inside a sunspot, however, this process does not occur since the magnetic field stops the granulation and makes the surface of the sunspot cooler and darker than the surrounding photosphere.

Observations of sunspots from Hinode and from the Swedish Solar Telescope on La Palma have revealed fine structure that would have delighted and intrigued Alfvén. Also, recent computational models have produced amazingly realistic images, which show that all the fine structure is a

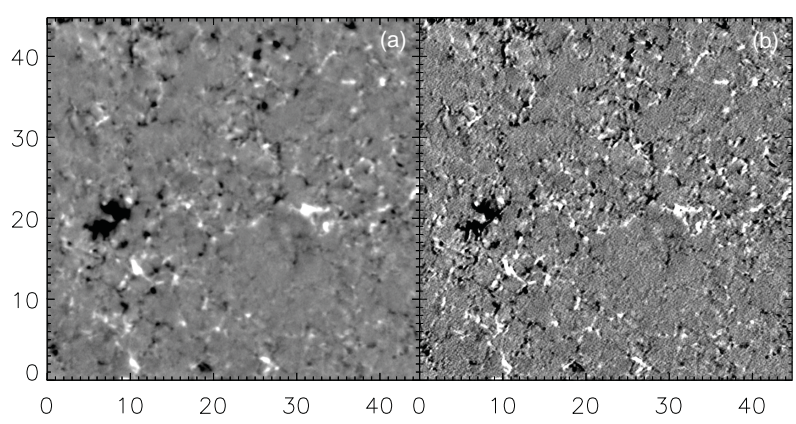

Figure 4. Line-of-sight magnetic field from (b) the IMAX instrument on the Sunrise balloon and (a) the same data at the resolution of the Hinode mission. The bipolar fragments (black and white next to one another) represent emerging flux if they are separating and cancelling flux if they are approaching. Distances are measured in arcsec, where 1 arcsec represents $726 \mathrm{~km}$ on the Sun (courtesy of Sami Solanki).

natural consequence of convection in the presence of a magnetic field (Rempel, 2012, 2015).

\subsection{The chromosphere}

The IRIS satellite has revealed that the chromosphere is very dynamic and has much fine structure. Spicules can be see at the limb of the Sun as cool jets of plasma that are ejected from the network at the edges of supergranules. They are heated to transition-region temperatures as they rise (Skogsrud et al., 2015; Carlsson et al., 2015) and are often twisted (De Pontieu et al., 2014), but their contribution to the solar wind is not yet known.

The Bifrost code has been developed in Oslo to model the complex transition through the chromosphere to the corona (Martínez-Sykora et al., 2009; Hansteen et al., 2010, 2015; Carlsson et al., 2010; Gudiksen et al., 2011). It has provided new insight into the nature of the complex connection between the photosphere and the corona, which involves transitions between the forces from being pressure dominated to those being magnetically dominated, the plasma from being neutral to being fully ionised, the radiation from being optically thick to being optically thin and the thermodynamic state from being in local equilibrium to being in non-equilibrium.

\section{Magnetic reconnection in three dimensions}

Before continuing to a discussion of coronal heating and solar flares, let us have a little detour to summarise the fundamental plasma process of magnetic reconnection, which is likely to be involved in both topics (Priest and Forbes, 2000). The basic theory for the process in two dimensions is now well understood, but there are key differences when it comes to three dimensions. 


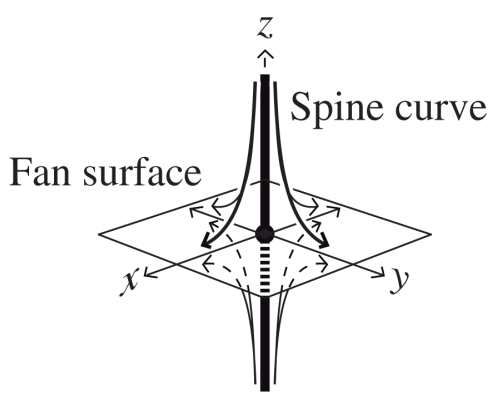

(a)



(b)
Figure 5. (a) The magnetic structure near a 3-D null point where the magnetic field vanishes. The spine is an isolated field line that approaches or recedes from the null point. (b) The field lines for a null point with magnetic components $\left(B_{x}, B_{y}, B_{z}\right)=(x, y,-2 z)$.

First of all, the magnetic structure near a null point, at which the magnetic field vanishes, is quite different in 2-D and 3-D. In 2-D a null point forms either an X-type or an Otype structure, but in 3-D the simplest field with a null at the origin and satisfying $\nabla \cdot \boldsymbol{B}=0$ has components

$$
\left(B_{x}, B_{y}, B_{z}\right)=(x, y,-2 z) \text {, }
$$

with field lines indicated in Fig. 5. Two families of field lines link to the null point: an isolated spine field line, which comes in along the positive and negative $z$ axis towards the null, and a surface of fan field lines, which comes out of the null in the $x-y$ plane. The fan surface is a so-called separatrix surface that separates the field lines that lie above the $x-y$ plane from those that lie below it.

Secondly, the topology in 3-D is much more complex than in 2-D. In 2-D, consider four sources in a line on the base (the photosphere) which are situated in the order,,,+-+(Fig. 6a). Above the base in the corona there is an X-type null point. The field lines that thread the null point are called separatrix curves, since they separate the 2-D region into topologically distinct subregions in the sense that the field lines immediately to the left of the null all start out at the leftmost positive source and end at the leftmost negative source; those to the right of the null link the other two sources. Furthermore, all the field lines below the null join the rightmost positive source to the leftmost negative source, while all the field lines above the null join the two outermost sources.

In 3-D, by comparison, suppose two positive and two negative sources are placed on the plane base (photosphere), as indicated in Fig. 6b. Two 3-D null points will be present on the base, indicated by large dots, and the fan surfaces from each of those null points form domes that curve downwards and separate the field lines below the dome from those above it. When the two domes intersect, they do so in a special curve called a separator that joins the one null point to the other (Fig. 7).

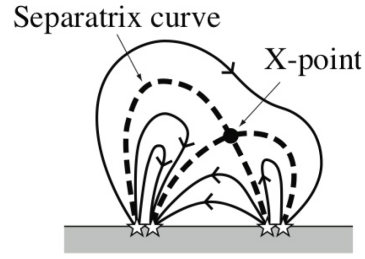

(a)

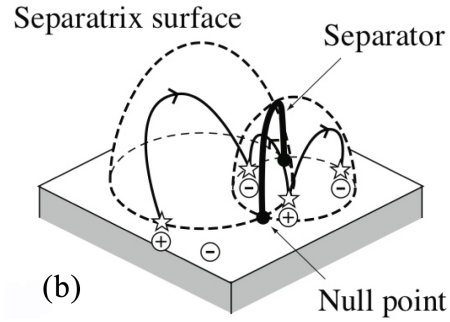

Figure 6. (a) The structure in 2-D near an X-type null point due to four sources on the base, showing how separatrix curves linking the $\mathrm{X}$-point separate the 2-D region into topologically separate regions. (b) The structure in 3-D due to four sources on the base, showing how separatrix surfaces linking two null points (also on the base) separate the 3-D region into topologically separate volumes. The two separatrices intersect in a special field line called a separator that joins the one null point to the other.

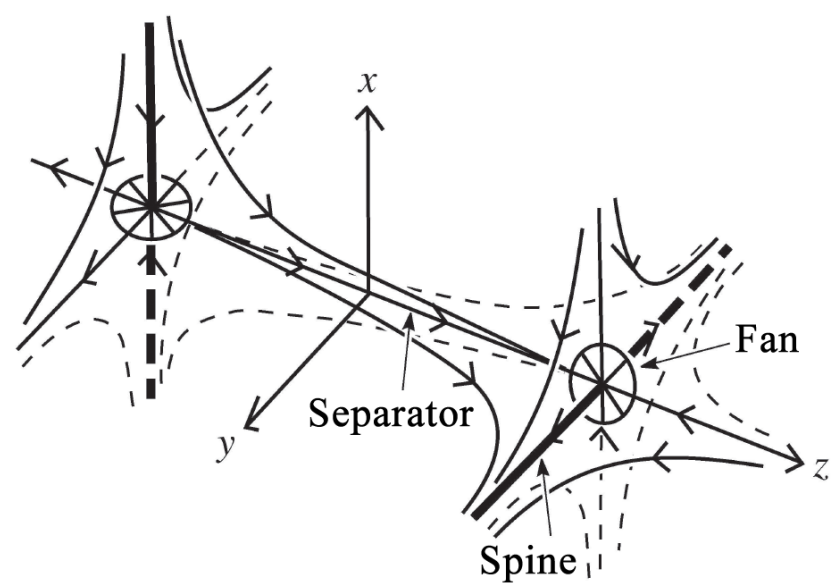

Figure 7. The magnetic structure near a separator lying along the $z$ axis and joining one null point to another.

The third difference between 2-D and 3-D lies in the nature of reconnection. In 2-D, reconnection can take place only at an X-point, and in Fig. 6a it transfers magnetic flux from two subregions to the other two subregions. In 3-D, one type of reconnection, called separator reconnection (Priest and Titov, 1996; Longcope and Cowley, 1996; Galsgaard and Nordlund, 1996; Longcope, 2001; Parnell and Galsgaard, 2004; Parnell et al., 2010), occurs when a strong current builds up along a separator. It has the effect of transferring magnetic flux from two regions to two other regions.

However, in 3-D, reconnection can also take place in other ways: at null points (Priest and Pontin, 2009), through torsional spine reconnection, torsional fan reconnection or, most usually, through spine-fan reconnection (Pontin et al., 2005); or quasi-separator reconnection when the current accumulates at a so-called quasi-separator (Priest and Démoulin, 1995; Démoulin et al., 1996; Titov et al., 2003; Aulanier et al., 2005). Consider the mapping of the footpoints of magnetic field lines from one part of the photosphere to another 
If there is a 3-D null point in the overlying atmosphere, then there is a separatrix surface across which the mapping exhibits a sudden jump or discontinuity. However, when there is no null point or separatrix surface, there are sometimes socalled quasi-separatrix surfaces, or QSLs for short, across which the mapping is perfectly continuous but possesses a steep gradient. Then large currents can once again accumulate along the quasi-separator and lead to magnetic reconnection.

An example of such a quasi-separator is shown in Fig. 8, for which the magnetic field just consists of an X-type nullpoint field in $x-y$ planes and a uniform field $\left(B_{0}\right)$ in the $z$ direction. The $z$ component of the field therefore removes the null points, but the separatrices that exist in the $x-y$ and $x-z$ planes when $B_{0}=0$ become quasi-separatrices when $B \neq 0$. Thus, for example, as a footpoint moves from $B_{1}$ a small distance to $B_{2}$, the other end of the field line flips very rapidly over a large distance from $A_{1}$ to $A_{2}$.

A final important new feature in three dimensions that we shall use in Sect. 6 is the existence of a topological invariant known as magnetic helicity, which includes two types: self-helicity and mutual helicity (Berger, 1984; Hornig and Schindler, 1996). The self-helicity is a measure of the twisting and kinking of a magnetic flux tube, whereas the mutual helicity measures the linkage between different flux tubes. What happens during 3-D reconnection is that the total magnetic helicity (the sum of self-helicity and mutual helicity) is conserved, but it can be converted from one kind to the other, for example, from mutual to self-helicity.

\section{The corona}

The corona has a temperature of about 1 million degrees $\mathrm{K}$, and in 1970 (apart from the occasional rocket flight) we could only glimpse it with one image per year from an eclipse observation. The corona was thought to be heated by sound waves generated by granulation, propagating upwards and then steepening to form shock waves.

Now, in addition to eclipse photographs we have regular images from artificial eclipses produced by space-borne coronagraphs and can also view the corona direct regularly in EUV and X-rays from space (Fig. 9). The corona has three different types of structure: small X-ray bright points lying above opposite-polarity magnetic fragments in the photosphere, coronal loops of many different types and coronal holes through which the magnetic field is open to interplanetary space and along which the fast solar wind escapes. The slow solar wind comes from near the equatorial streamer belt.

Key unanswered questions are related to the nature of coronal heating (which is now known to be magnetic), the acceleration of the solar wind and the nature of huge magnetic flux ropes called prominences, which lie in the corona but have chromospheric temperatures. Prominences lie above polarity inversion lines, at which the line-of-sight magnetic



Figure 8. The magnetic structure near a quasi-separator located along the $z$ axis.

field changes polarity. Occasionally, they erupt outwards in association with and lying in the core of coronal mass ejections. When they erupt from active regions, they also produce solar flares.

\subsection{Prominences}

Recently, an impressive non-linear force-free model of the coronal magnetic field has been set up (Mackay and van Ballegooijen, 2006; Mackay and Yeates, 2012; Yeates et al., 2008; Yeates and Mackay, 2009). It evolves through a series of equilibria in response to the observed surface motions at the Sun: differential rotation, meridional flow, flux diffusion and emergence of new flux (Fig. 10a). Along certain portions of the global polarity inversion line, twisted magnetic flux ropes appear; at the same locations, $H \alpha$ maps reveal the presence of prominences. The locations of the vast majority of prominences that are either quiescent (i.e., outside active regions) or intermediate (i.e., at the boundaries of active regions) can be predicted with the code (Fig. 10b). Furthermore, the timing for the eruption of such prominences can be predicted to within 1 day.

\subsection{Coronal heating}

The coronal heating problem probably has a variety of different solutions in different parts of the solar atmosphere. Thus, coronal holes and the high corona are likely to be heated by magnetic waves propagating from below and dissipating by phase mixing or resonant absorption (Heyvaerts and Priest, 1983; Tomczyk and McIntosh, 2009; Nakariakov et al., 1999; Goossens et al., 2011; De Moortel and Nakariakov, 2012), with the dissipation mechanism being a collisionless one. Xray bright points, on the other hand, are almost certainly produced directly by magnetic reconnection, since they lie above opposite-polarity magnetic sources that either move apart as 

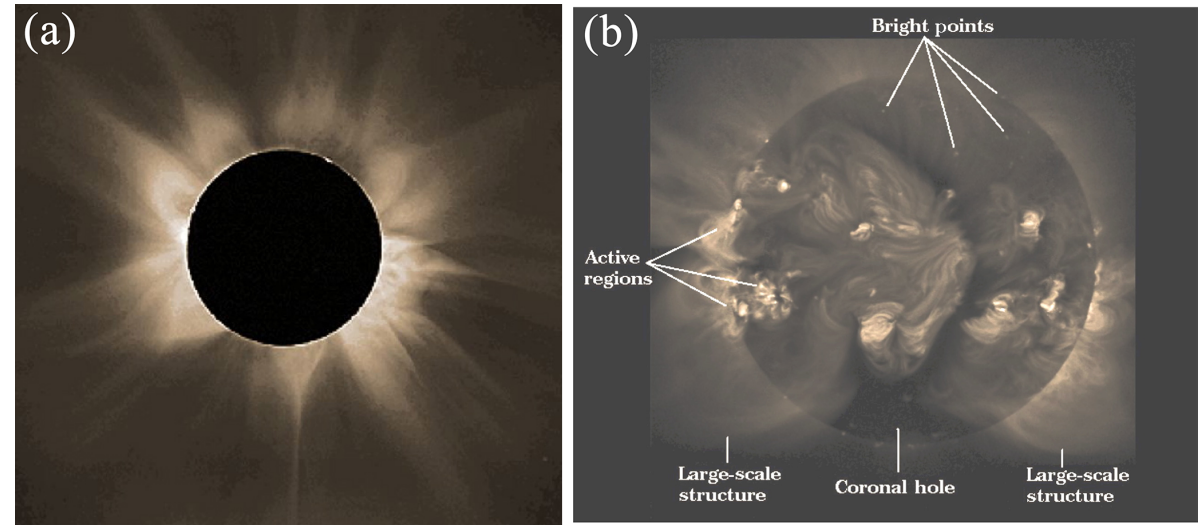

Figure 9. The solar corona as viewed (a) in white light during an eclipse near solar maximum, courtesy of the High Altitude Observatory (HAO), University Corporation for Atmospheric Research (UCAR) in Boulder, Colorado. UCAR is sponsored by the National Science Foundation. (b) The solar corona in soft X-rays from the Yohkoh satellite (courtesy of Saku Tsuneta).
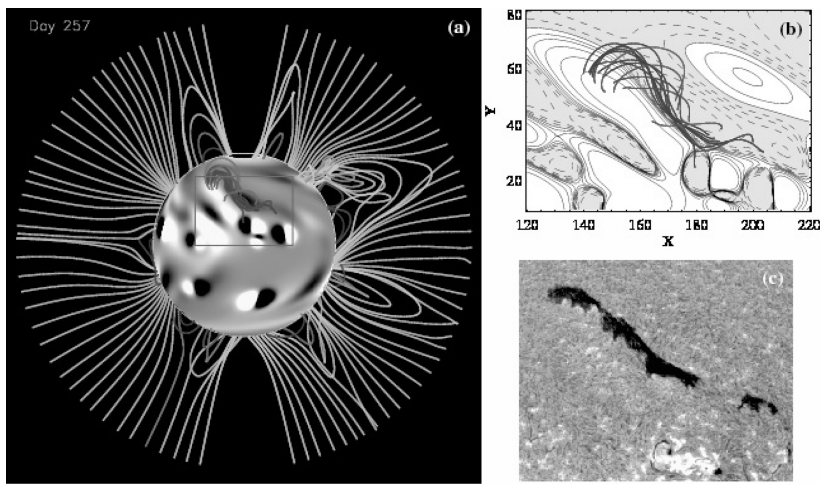

Figure 10. (a) A non-linear force-free model of the solar corona from a global simulation after 108 days of evolution, showing highly twisted flux ropes, weakly sheared arcades and near-potential open fields. (b) A close-up showing a twisted flux rope lying above part of the polarity inversion line and (c) the associated solar prominence observed by Big Bear Solar Observatory in $H \alpha$ (courtesy of Duncan Mackay).

new flux emerges and reconnects with the overlying field or come closer together and drive reconnection as they cancel (Priest et al., 1994; Parnell and Priest, 1995). MHD turbulence models may be used to describe the cascade of energy from large to small scales and its dissipation.

The coronal tectonics model (Priest et al., 2002) is a refinement of Parker's braiding and his nano-flare model. Coronal tectonics takes account of the fact that the magnetic field of the corona comes through the solar surface from many small intense photospheric sources rather than through regions of weak uniform magnetic field. Thus, the flux from the sources spreads out in a highly complex way into the overlying atmosphere, and the fluxes from neighbouring sources are separated from one another by a complex web of separatrix surfaces (Fig. 11). Thus, the response to the complex motions of these sources is the formation of a myriad of current sheets

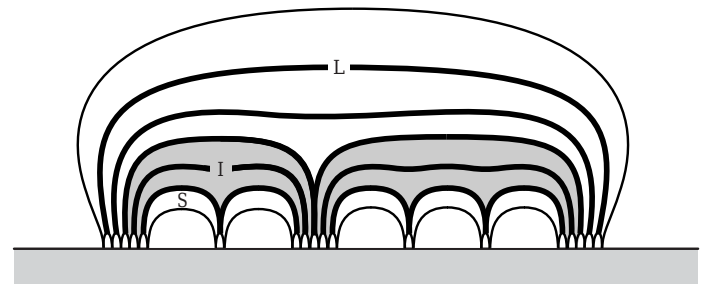

Figure 11. The coronal tectonics model, showing a schematic of a coronal loop consisting of many sub-volumes, each linked to a separate photospheric source and divided from one another by separatrix surfaces. The motions of the footpoints produce a myriad of current sheets along the separatrices, which dissipate impulsively and produce nano-flares.

along the separatrices. These current sheets continually form and dissipate through magnetic reconnection and produce a complex web of impulsive nano-flares that are highly likely to be heating the corona, especially in active regions and in other types of coronal loop.

\subsection{The solar wind}

In 1970, the focus was simply on Parker models of the solar wind, with the flow velocity $(v(r))$ being spherically symmetric and a function only of distance $(r)$ from the Sun. Now the models are highly sophisticated, and observations from the Ulysses mission have revealed that at sunspot minimum there is a mixture of fast solar wind at $700 \mathrm{~km} \mathrm{~s}^{-1}$ from coronal holes and much more sporadic slow solar wind from streamer belts. On the other hand, at sunspot maximum the solar wind is much more irregular (Fig. 12).

The fast solar wind is likely to originate in coronal funnels, as shown in Hinode observations of outflows (Sakao et al., 2007). Models of the magnetic topology of the corona have revealed a series of funnels bounded by open separatrices and often spreading out from small locations at the solar surface 


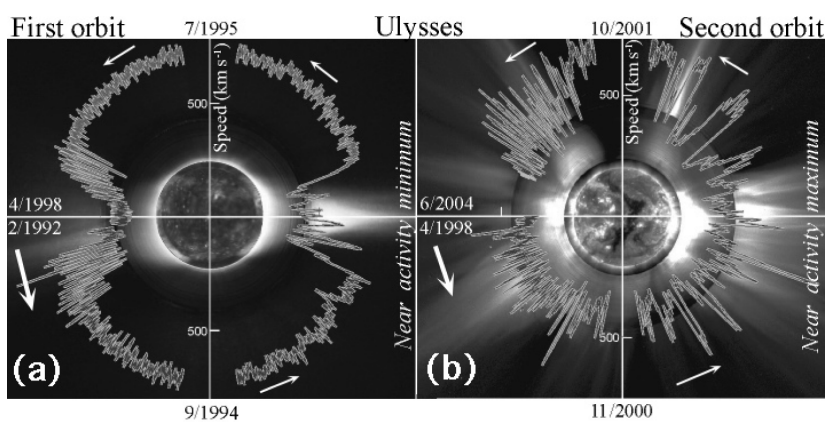

Figure 12. The speed of the solar wind as a function of latitude in polar coordinates from the Ulysses spacecraft during (a) solar minimum and (b) solar maximum. Superimposed are coronal images from the Mauna Loa K coronameter, plus the EIT and LASCO instruments on the Solar Dynamics Observatory (SDO) (from MeyerVernet, 2007).

(Platten et al., 2014; Edwards et al., 2016). So the fast wind is in my view likely to be heated by reconnection low down in funnels and then by a turbulent cascade of MHD waves higher up (Cranmer, 2012). On the other hand, the mechanism for accelerating the slow solar wind is much more uncertain. One possibility is that it is heated by the equivalent of the tectonics model, but operating in a web of separatrices near streamer boundaries (the so-called $S$-web) (Linker et al., 2011; Titov et al., 2011).

\section{Solar flares and coronal mass ejections}

In 1970, a solar flare was defined as "a brightening in the chromosphere", and coronal mass ejections (CMEs) had not been born. Now we know that the core of a flare is located in the corona and involves the eruption of a coronal magnetic flux tube, which drives reconnection below it. The magnetic flux tube is revealed as an erupting prominence and the surrounding coronal structure becomes a CME (Fig. 13).

A schematic of the main phase of the overall process is shown in Fig. 14a; a vertical cross section through it (Fig. 14b) indicates the nature of the reconnection that is driven below the erupting prominence. The heat and fast particles that are produced during the reconnection propagate down to the feet of the reconnected field lines and drive chromospheric upflows, which fill a coronal loop with hot dense plasma through a process called chromospheric evaporation. At the base of the resulting hot coronal arcade, one sees a pair of bright chromospheric ribbons. As the reconnection proceeds, the location of the reconnection rises in the atmosphere, so new higher coronal loops are heated (Fig. 14c) while the ribbons separate in time. Meanwhile, the previous coronal loops cool through radiation and heat conduction. The reconnection adds both magnetic flux and magnetic helicity to the erupting flux rope.
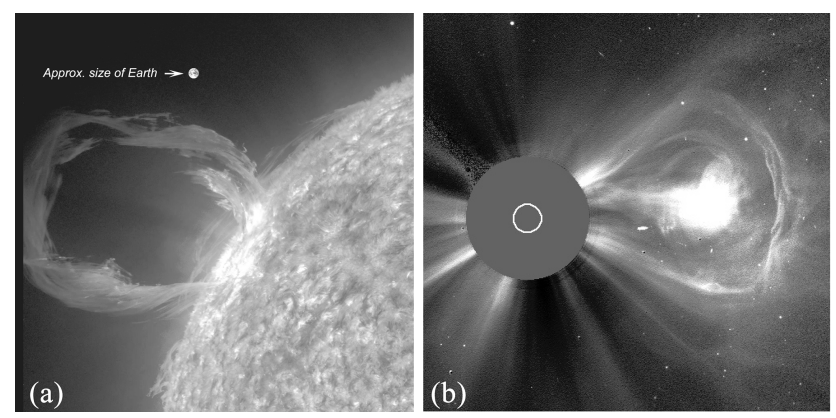

Figure 13. (a) An erupting prominence in He II 304 with the size of the Earth indicated (from the AIA instrument on SDO). (b) A coronal mass ejection observed by the LASCO instrument on-board SOHO.
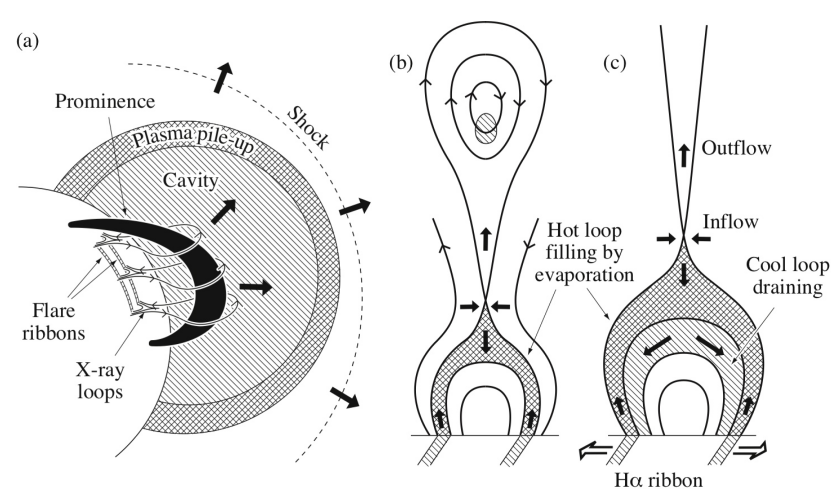

Figure 14. (a) Schematics of a solar flare, showing (a) the 3-D structure and (b) a vertical cross section early in the main phase and (c) later on.

The cause of the eruption may be a lack of equilibrium, called non-equilibrium, or it may be a torus or kink instability. It appears that in some flares, separator reconnection is at work, while others may be focussed on quasi-separators or on coronal null points. Some of the twist that is observed in the erupting flux rope and further out in magnetic clouds may come from an initial flux tube located below the coronal arcade before it erupts. However, we have just suggested a new process that may create most of the twist (Priest and Longcope, 2017).

During the rise phase of a flare, there are chromospheric brightenings at small points on either side of the polarity inversion line (PIL), which then spread along the PIL to create the ribbons. We suggest, therefore, that the initial sheared arcade (overlying an initial small flux rope $Z_{+} Z_{-}$) consists of a series of flux tubes $A_{+} A_{-}, B_{+} B_{-}, C_{+} C_{-}$and $D_{+} D_{-}$ (Fig. 15a). The flare in our model spreads along the PIL by so-called zipper reconnection, in which $A_{+} A_{-}$first reconnects with $B_{+} B_{-}$(Fig. 15b), then with $C_{+} C_{-}$(Fig. 15c) and finally with $D_{+} D_{-}$(Fig. 15d).

At each reconnection, the total magnetic helicity is conserved, but the mutual magnetic helicity of the sheared (in- 

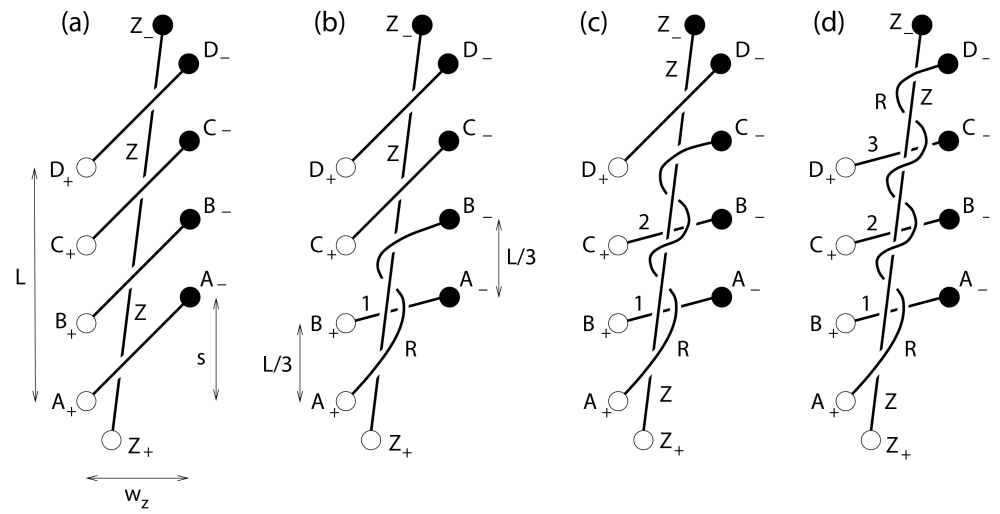

Figure 15. Zipper reconnection in a coronal arcade, seen from above, during the rise phase of a flare or coronal mass ejection. (a) Suppose the initial arcade consists of four parallel flux tubes connecting sources $A_{+} \rightarrow A_{-}, B_{+} \rightarrow B_{-}, C_{+} \rightarrow C_{-}$and $D_{+} \rightarrow D_{-}$. These overlie a pre-existing flux rope $Z_{+} Z_{-}$. The zipper reconnection sequence consists of three individual reconnection events (b-d). The overlying twisted flux tube is designated $R$, and the part of the reconnected arcade tube that is generated in reconnection event $n$ is designated with that number, i.e., 1,2 or 3.

ner) arcade is converted into self-helicity of the new flux rope. Thus, after the first reconnection, the reconnected field line $A_{+} B_{-}$is twisted about the initial tube $Z_{+} Z_{-}$. After several reconnections, the end effect of the zipper process is to create a core of high twist $A_{+} D_{-}$around the initial tube.

During the main phase of the flare, the flux rope continues to grow by quasi-2-D reconnection, with mutual helicity of the outer coronal arcade being converted to self-helicity of the outer part of the flux rope (Fig. 16). The net effect is that the final erupting flux rope consists of three parts: a central inner core that was present initially, a highly twisted outer core that was created by zipper reconnection during the rise phase and an outer less-twisted sheath created during the main phase.

\section{Conclusions}

Solar physics is at an extremely exciting phase, since there are many intriguing, fundamental and unsolved problems. Huge progress has been made over the past 10 years which would have delighted Alfvén, but definitive detailed answers have not yet been provided about the following:

- how the solar magnetic field is generated;

- how the dynamic fine-scale structure of the solar atmosphere is created;

- how the different parts of the corona are heated;

- how the different types of solar wind are accelerated;

- how eruptive solar flares and coronal mass ejections are initiated;

- and how reconnection converts magnetic energy to kinetic energy, heat and fast-particle energy in flares.

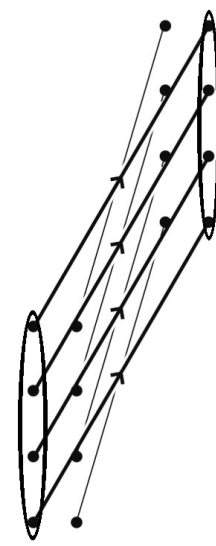

(a)

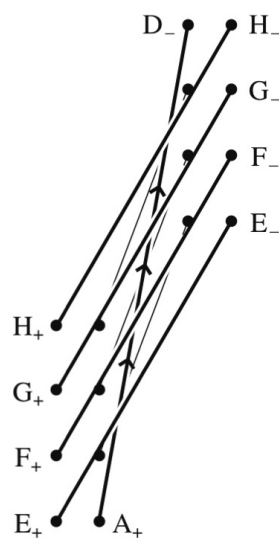

(b)

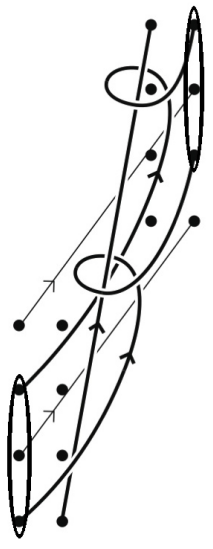

(c)
Figure 16. Quasi-2-D reconnection during the main phase of a flare or coronal mass ejection. (a) The initial sheared state. (b) The situation after the zipper phase of the flare when reconnection has proceeded parallel to the polarity inversion line to produce the zipper flux rope $\left(A_{+} D_{-}\right)$lying under the next set of field lines joining footpoints $E_{+} E_{-}, F_{+} F_{-}, G_{+} G_{-}, H_{+} H_{-}$. (c) The first part of the main phase after reconnection has progressed sideways to reconnect the next sheath of field lines and create a spiral sheath that wraps around the zipper flux rope and enhances its flux and magnetic helicity.

Future breakthroughs will come from a combination of new observations, especially from the European Solar Orbiter mission (2019) and the DKIST $4 \mathrm{~m}$ telescope on Hawaii, but also from clever new ideas and models in the spirit of Hannes Alfvén. So, in the meantime let us relish the challenge and enjoy the beauty of the Sun. 
Data availability. No data sets were used in this article.

Competing interests. The author declares that he has no conflict of interest.

Acknowledgements. I am delighted to acknowledge the fun that many collaborators, colleagues, students and postdocs have given me as we have shared ideas over the years. Without them and the unceasing support of my wife, Clare, and my family I would not have been able to embark on the many research journeys.

The topical editor, Manuela Temmer, thanks two anonymous referees for help in evaluating this paper.

\section{References}

Archontis, V. and Hansteen, V.: Clusters of Small Eruptive Flares Produced by Magnetic Reconnection in the Sun, Astrophys. J. Lett., 788, L2, https://doi.org/10.1088/2041-8205/788/1/L2, 2014.

Archontis, V., Hood, A. W., and Tsinganos, K.: Recurrent Explosive Eruptions and the "Sigmoid-to-arcade" Transformation in the Sun Driven by Dynamical Magnetic Flux Emergence, Astrophys. J. Lett., 786, L21, https://doi.org/10.1088/2041-8205/786/2/L21, 2014.

Aulanier, G., Pariat, E., and Démoulin, P.: Current-sheet formation in quasi-separatrix layers and hyperbolic flux tubes, Astron. Astrophys., 444, 961-976, https://doi.org/10.1051/00046361:20053600, 2005.

Berger, M. A.: Rigorous new limits on magnetic helicity dissipation in the solar corona, Geophys. Astrophys. Fluid Dynam., 30, 79104, https://doi.org/10.1080/03091928408210078, 1984.

Birkeland, K.: The Norwegian Aurora Polaris Expedition, 19021903, in: vol. I, Sect. 1, H. Aschehoug, Oslo, 1908.

Carlsson, M., Hansteen, V. H., and Gudiksen, B. V.: Chromospheric heating and structure as determined from high resolution 3D simulations, Mem. della Soc. Astron. Ital., 81, 582-587, 2010.

Carlsson, M., Leenaarts, J., and De Pontieu, B.: What Do IRIS Observations of $\mathrm{Mg}$ II $\mathrm{k}$ Tell Us about the Solar Plage Chromosphere?, Astrophys. J. Lett., 809, L30, https://doi.org/10.1088/2041-8205/809/2/L30, 2015.

Chapman, S. and Ferraro, V. C. A.: A New Theory of Magnetic Storms, Nature, 126, 129-130, https://doi.org/10.1038/126129a0, 1930.

Christensen-Dalsgaard, J., Duvall, T. L., Gough, D. O., Harvey , J. W., and Rhodes, E. J.: Speed of sound in the solar interior, Nature, 315, 378-382, https://doi.org/10.1038/315378a0, 1985.

Cranmer, S. R.: Self-Consistent Models of the Solar Wind, Space Sci. Rev., 172, 145-156, https://doi.org/10.1007/s11214-0109674-7, 2012.

De Moortel, I. and Nakariakov, V. M.: Magnetohydrodynamic waves and coronal seismology: an overview of recent results, Philos. T. Roy. Soc. Lond. A, 370, 3193-3216, https://doi.org/10.1098/rsta.2011.0640, 2012.

De Pontieu, B., Rouppe van der Voort, L., McIntosh, S. W., Pereira, T. M. D., Carlsson, M., Hansteen, V., Skogsrud, H., Lemen, J., Title, A., Boerner, P., Hurlburt, N., Tarbell, T. D., Wuelser, J.
P., De Luca, E. E., Golub, L., McKillop, S., Reeves, K., Saar, S., Testa, P., Tian, H., Kankelborg, C., Jaeggli, S., Kleint, L., and Martinez-Sykora, J.: On the prevalence of small-scale twist in the solar chromosphere and transition region, Science, 346, 1255732, https://doi.org/10.1126/science.1255732, 2014.

Démoulin, P., Henoux, J., Priest, E. R., and Mandrini, C. H.: Quasiseparatrix layers in solar flares. I. Method, Astron. Astrophys., 308, 643-655, 1996.

Dungey, J.: Interplanetary magnetic field and the auroral zones, Phys. Rev. Lett., 6, 47-48, https://doi.org/10.1103/PhysRevLett.6.47, 1961.

Duvall, T. L. and Harvey, J. W.: Rotational frequency splitting of solar oscillations, Nature, 310, 19-22, https://doi.org/10.1038/310019a0, 1984.

Duvall, T. L., Dziembowski, W. A., Goode, P. R., Gough, D. O., Harvey, J. W., and Leibacher, J. W.: Internal rotation of the Sun, Nature, 310, 22-25, https://doi.org/10.1038/310022a0, 1984.

Edwards, S. J., Parnell, C. E., Harra, L. K., Culhane, J. L., and Brooks, D. H.: A Comparison of Global Magnetic Field Skeletons and Active-Region Upflows, Solar Phys., 291, 117-142, https://doi.org/10.1007/s11207-015-0807-8, 2016.

Galsgaard, K. and Nordlund, A.: The heating and activity of the solar corona: I Boundary shearing of an initially homogeneous magnetic field, J. Geophys. Res., 101, 13445-13460, https://doi.org/10.1029/96JA00428, 1996.

Goossens, M., Erdélyi, R., and Ruderman, M. S.: Resonant MHD Waves in the Solar Atmosphere, Space Sci. Rev., 158, 289-338, https://doi.org/10.1007/s11214-010-9702-7, 2011.

Gudiksen, B. V., Carlsson, M., Hansteen, V. H., Hayek, W., Leenaarts, J., and Martínez-Sykora, J.: The stellar atmosphere simulation code Bifrost. Code description and validation, Astron. Astrophys., 531, A154, https://doi.org/10.1051/00046361/201116520, 2011.

Hansteen, V. H., Hara, H., De Pontieu, B., and Carlsson, M.: On Redshifts and Blueshifts in the Transition Region and Corona, Astrophys. J., 718, 1070-1078, https://doi.org/10.1088/0004637X/718/2/1070, 2010.

Hansteen, V. H., Guerreiro, N., De Pontieu, B., and Carlsson, M.: Numerical Simulations of Coronal Heating through Footpoint Braiding, Astrophys. J., 811, 106, https://doi.org/10.1088/0004637X/811/2/106, 2015.

Hansteen, V. H., Archontis, V., Pereira, T. M. D., Carlsson, M., Rouppe van der Voort, L., and Leenaarts, J.: Bombs and Flares at the Surface and Lower Atmosphere of the Sun, Astrophys. J., 839, 22, https://doi.org/10.3847/1538-4357/aa6844, 2017.

Heyvaerts, J. and Priest, E. R.: Coronal heating by phase-mixed shear Alfvén waves, Astron. Astrophys., 117, 220-234, 1983.

Hornig, G. and Schindler, K.: Magnetic topology and the problem of its invariant definition, Phys. Plasmas, 3, 781-791, https://doi.org/10.1063/1.871778, 1996.

Linker, J. A., Lionello, R., Mikić, Z., Titov, V. S., and Antiochos, S. K.: The Evolution of Open Magnetic Flux Driven by Photospheric Dynamics, Astrophys. J., 731, 110, https://doi.org/10.1088/0004-637X/731/2/110, 2011.

Longcope, D. W.: Separator current sheets: Generic features in minimum-energy magnetic fields subject to flux constraints, Phys. Plasmas, 8, 5277-5289, https://doi.org/10.1063/1.1418431, 2001. 
Longcope, D. W. and Cowley, S. C.: Current sheet formation along three-dimensional magnetic separators, Phys. Plasmas, 3, 28852897, https://doi.org/10.1063/1.871627, 1996.

Mackay, D. H. and van Ballegooijen, A. A.: Models of the Large-Scale Corona. I. Formation, Evolution, and Liftoff of Magnetic Flux Ropes, Astrophys. J., 641, 577-589, https://doi.org/10.1086/500425, 2006

Mackay, D. H. and Yeates, A.: The Sun's Global Photospheric and Coronal Magnetic Fields: Observations and Models, Liv. Rev. Sol. Phys., 9, 6, https://doi.org/10.12942/lrsp-2012-6, 2012.

Martínez-Sykora, J., Hansteen, V., DePontieu, B., and Carlsson, M.: Spicule-Like Structures Observed in Three-Dimensional Realistic Magnetohydrodynamic Simulations, Astrophys. J., 701, 1569-1581, https://doi.org/10.1088/0004-637X/701/2/1569, 2009.

Martínez-Sykora, J., Moreno-Insertis, F., and Cheung, M. C. M.: Multi-parametric Study of Rising 3D Buoyant Flux Tubes in an Adiabatic Stratification Using AMR, Astrophys. J., 814, 2, https://doi.org/10.1088/0004-637X/814/1/2, 2015.

Meyer-Vernet, N.: Basics of the Solar Wind, Cambridge University Press, Cambridge, UK, 2007.

Nakariakov, V. M., Ofman, L., DeLuca, E. E., Roberts, B., and Davila, J. M.: TRACE observation of damped coronal loop oscillations: Implications for coronal heating, Science, 285, 862-864, https://doi.org/10.1126/science.285.5429.862, 1999.

Parnell, C. E. and Galsgaard, K.: Elementary Heating Events - Interaction between Two Flux Sources. II Rates of Flux Reconnection, Astron. Astrophys., 428, 595-612, https://doi.org/10.1051/0004-6361:20034350, 2004.

Parnell, C. E. and Priest, E. R.: A converging flux model for the formation of an X-ray bright point above a supergranule cell, Geophys. Astrophys. Fluid Dynam., 80, 255-276, https://doi.org/10.1080/03091929508228958, 1995.

Parnell, C. E., Haynes, A. L., and Galsgaard, K.: Structure of magnetic separators and separator reconnection, J. Geophys. Res., 115, 2102, https://doi.org/10.1029/2009JA014557, 2010.

Platten, S. J., Parnell, C. E., Haynes, A. L., Priest, E. R., and Mackay, D. H.: The solar cycle variation of topological structures in the global solar corona, Astron. Astrophys., 565, A44, https://doi.org/10.1051/0004-6361/201323048, 2014.

Pontin, D. I., Galsgaard, K., Hornig, G., and Priest, E. R.: A fully magnetohydrodynamic simulation of 3D non-null reconnection, Phys. Plasmas, 12, 052307, https://doi.org/10.1063/1.1891005, 2005

Priest, E. R. and Démoulin, P.: Three-dimensional magnetic reconnection without null points. 1 Basic theory of magnetic flipping, J. Geophys. Res., 100, 23443-23463, https://doi.org/10.1029/95JA02740, 1995.

Priest, E. R. and Forbes, T. G.: Magnetic Reconnection: MHD Theory and Applications, Cambridge University Press, Cambridge, UK, 2000

Priest, E. R. and Longcope, D. W.: Flux-Rope Twist in Eruptive Flares and CMEs: Due to Zipper and Main-Phase Reconnection, Solar Phys., 292, 25, https://doi.org/10.1007/s11207-016-10490, 2017.

Priest, E. R. and Pontin, D. I.: Three-dimensional null point regimes, Phys. Plasmas, 16, 122101, https://doi.org/10.1063/1.3257901, 2009.
Priest, E. R. and Titov, V. S.: Magnetic reconnection at threedimensional null points, Philos. T. Roy. Soc. Lond., 355, 29512992, https://doi.org/10.1098/rsta.1996.0136, 1996.

Priest, E. R., Parnell, C. E., and Martin, S. F.: A converging flux model of an X-ray bright point and an associated canceling magnetic feature, Astrophys. J., 427, 459-474, https://doi.org/10.1086/174157, 1994.

Priest, E. R., Heyvaerts, J., and Title, A.: A Flux Tube Tectonics model for solar coronal heating driven by the magnetic carpet, Astrophys. J., 576, 533-551, https://doi.org/10.1086/341539, 2002.

Rempel, M.: Numerical Sunspot Models: robustness of Photospheric Velocity and Magnetic Field Structure, Astrophys. J., 750, 62, https://doi.org/10.1088/0004-637X/750/1/62, 2012.

Rempel, M.: Numerical Simulations of Sunspot Decay: On the Penumbra-Evershed Flow-Moat Flow Connection, Astrophys. J., 814, 125, https://doi.org/10.1088/0004-637X/814/2/125, 2015.

Sakao, T., Kano, R., Narukage, N., Kotoku, J., Bando, T., DeLuca, E. E., Lundquist, L. L., Tsuneta, S., Harra, L. K., Katsukawa, Y., Kubo, M., Hara, H., Matsuzaki, K., Shimojo, M., Bookbinder, J. A., Golub, L., Korreck, K. E., Su, Y., Shibasaki, K., Shimizu, T., and Nakatani, I.: Continuous Plasma Outflows from the Edge of a Solar Active Region as a Possible Source of Solar Wind, Science, 318, 1585-1588, https://doi.org/10.1126/science.1147292, 2007.

Schou, J., Antia, H. M., Basu, S., Bogart, R. S., Bush, R. I., Chitre, S. M., Christensen-Dalsgaard, J., di Mauro, M. P., Dziembowski, W. A., Eff-Darwich, A., Gough, D. O., Haber, D. A., Hoeksema, J. T., Howe, R., Korzennik, S. G., Kosovichev, A. G., Larsen, R. M., Pijpers, F. P., Scherrer, P. H., Sekii, T., Tarbell, T. D., Title, A. M., Thompson, M. J., and Toomre, J.: Helioseismic Studies of Differential Rotation in the Solar Envelope by the Solar Oscillations Investigation Using the Michelson Doppler Imager, Astrophys. J., 505, 390-417, https://doi.org/10.1086/306146, 1998.

Skogsrud, H., Rouppe van der Voort, L., De Pontieu, B., and Pereira, T. M. D.: On the Temporal Evolution of Spicules Observed with IRIS, SDO, and Hinode, Astrophys. J., 806, 170, https://doi.org/10.1088/0004-637X/806/2/170, 2015.

Solanki, S. K.: Overview of the Special Issue on the First Science Results from the Second Flight of Sunrise, Ap. J. Suppl., 229, 1, https://doi.org/10.3847/1538-4365/aa6742, 2017.

Solanki, S. K., Barthol, P., Danilovic, S., Feller, A., Gandorfer, A., Hirzberger, J., Riethmüller, T. L., Schüssler, M., Bonet, J. A., Martínez Pillet, V., del Toro Iniesta, J. C., Domingo, V., Palacios, J., Knölker, M., Bello González, N., Berkefeld, T., Franz, M., Schmidt, W., and Title, A. M.: SUNRISE: Instrument, Mission, Data, and First Results, Astrophys. J. Lett., 723, L127-L133, https://doi.org/10.1088/2041-8205/723/2/L127, 2010.

Solanki, S. K., Riethmüller, T. L., Barthol, P., Danilovic, S., Deutsch, W., Doerr, H.-P., Feller, A., Gandorfer, A., Germerott, D., Gizon, L., Grauf, B., Heerlein, K., Hirzberger, J., Kolleck, M., Lagg, A., Meller, R., Tomasch, G., van Noort, M., Blanco Rodríguez, J., Gasent Blesa, J. L., Balaguer Jiménez, M., Del Toro Iniesta, J. C., López Jiménez, A. C., Orozco Suarez, D., Berkefeld, T., Halbgewachs, C., Schmidt, W., Álvarez-Herrero, A., Sabau-Graziati, L., Pérez Grande, I., Martínez Pillet, V., Card, G., Centeno, R., Knölker, M., and Lecinski, A.: The Second Flight of the Sunrise Balloon-borne Solar Observatory: Overview of Instrument Updates, the Flight, the Data, and First 
Results, Ap. J. Supplement, 229, 2, https://doi.org/10.3847/15384365/229/1/2, 2017

Southwood, D.: From the Carrington Storm to the Dungey Magnetosphere, in: Magnetospheric Plasma Physics: The Impact of Jim Dungey's Research, vol. 41 of Astrophysics and Space Science Proceedings, edited by: Southwood, D., Cowley, S. W. H., and Mitton, S., p. 253, https://doi.org/10.1007/978-3-319-183596_12, Springer International Publishing Switzerland, 2015.

Titov, V. S., Galsgaard, K., and Neukirch, T.: Magnetic pinching of hyperbolic flux tubes. I. Basic estimations, Astrophys. J., 582, 1172-1189, https://doi.org/10.1086/344799, 2003.

Titov, V. S., Mikić, Z., Linker, J. A., Lionello, R., and Antiochos, S. K.: Magnetic Topology of Coronal Hole Linkages, Astrophys. J., 731, 111, https://doi.org/10.1088/0004-637X/731/2/111, 2011.
Tomczyk, S. and McIntosh, S. W.: Time-Distance Seismology of the Solar Corona with CoMP, Astrophys. J., 697, 1384-1391, https://doi.org/10.1088/0004-637X/697/2/1384, 2009.

Yeates, A. R. and Mackay, D. H.: Initiation of Coronal Mass Ejections in a Global Evolution Model, Astrophys. J., 699, 1024 1037, https://doi.org/10.1088/0004-637X/699/2/1024, 2009.

Yeates, A. R., Mackay, D. H., and van Ballegooijen, A. A.: Evolution and Distribution of Current Helicity in FullSun Simulations, Astrophys. J. Lett., 680, L165-L168, https://doi.org/10.1086/590057, 2008. 